\title{
IMportance of an Irregular Z Line: Is Barrett's in the Eye of the Beholder?
}

\author{
Swathi Eluri ${ }^{1} \cdot$ Nicholas J. Shaheen ${ }^{1}$
}

Published online: 28 February 2018

○) Springer Science+Business Media, LLC, part of Springer Nature 2018

\section{A painting only lives in the eye of the beholder.}

\section{-Pablo Picasso}

The $\mathrm{Z}$ line demarcates the squamocolumnar junction (SCJ), the transition from the squamous esophageal mucosa to the columnar mucosa lining the stomach, or in cases of Barrett's esophagus, between the squamous and intestinalized columnar epithelium of the metaplastic segment. An irregular $\mathrm{Z}$ line is characterized by $<1 \mathrm{~cm}$ columnar tongues that extend proximal to the gastroesophageal junction, a finding that has been reported in approximately $10-15 \%$ of the population undergoing upper endoscopy $[1,2]$. In those with an irregular $\mathrm{Z}$ line, there is up to a $44 \%$ prevalence of intestinal metaplasia (IM) [3]. Nevertheless, the clinical significance of this finding with respect to the risk of progression to neoplasia in patients with an irregular $\mathrm{Z}$ line and IM is unclear [4].

While prior guidelines are in conflict with regard to the management strategies for putative Barrett's esophagus (BE) segments $<1 \mathrm{~cm}$, the most recent American College of Gastroenterology (ACG) guidelines [5] recommend against obtaining biopsies from an irregular $\mathrm{Z}$ line, a recommendation made due to the poor endoscopic standardization of this finding (one endoscopist's normal $\mathrm{Z}$ line is another's irregular $\mathrm{Z}$ line), the additional costs associated with this practice, the potential for patient anxiety, and the low rates of prevalent and incident dysplasia and esophageal adenocarcinoma (EAC) found in these lesions. Also, patients with an irregular $\mathrm{Z}$ line who harbor IM might be enrolled in endoscopic surveillance protocols, necessitating the costs and inconvenience of recurrent surveillance endoscopy, as

Swathi Eluri

swathi@med.unc.edu

1 Center for Esophageal Diseases and Swallowing, Division of Gastroenterology and Hepatology, University of North Carolina, 4119B Bioinformatics Building, 130 Mason Farm Rd, Chapel Hill, NC 27599-7080, USA well as the stigma of a disease label for a potentially neoplastic condition.

A prospective single-center study by Itskoviz et al. published in the current issue of Digestive Diseases and Sciences [6] describes the risk of developing $\mathrm{BE}$, dysplasia, and EAC in those with an irregular $Z$ line and IM compared to those with an irregular $Z$ line without IM. There were 166 patients endoscopically diagnosed with an irregular $Z$ line, defined here as variability of $\leq 5 \mathrm{~mm}$ of the squamocolumnar junction on index endoscopy. Of the 166 patients who underwent upper endoscopy for indications such as heartburn, dysphagia, and chest pain, 39\% $(n=64)$ had biopsy-proven IM. Of the 50 with IM that were followed up for a median 70 months, $16 \%$ developed non-dysplastic $\mathrm{BE}(\mathrm{NDBE})(n=8)$, and $4 \%(n=2)$ developed low-grade dysplasia (LGD). Of the cases without IM, only $1 / 52$ developed $\mathrm{BE}$ and none progressed to dysplasia. In this sample, $22 \%$ of the subjects with IM and $49 \%$ without IM were lost to follow-up.

Overall, among patients with an irregular $\mathrm{Z}$ line in this study, $8.8 \%$ developed NDBE and $1.9 \%$ developed LGD over a median time of 70 months. The authors note in their conclusion that patients with an irregular Z line "do not develop major BE complication in more than 5 years of follow-up." Yet, they also suggest in their discussion that, due to the $16 \%$ incidence of BE in the IM-positive group and the two patients who developed LGD, "longer follow-up studies may reveal a more malicious nature of irregular $\mathrm{Z}$ line with intestinal metaplasia."

This study has several commendable strengths: It is prospective, with a standardized biopsy protocol with followup, at a median of 70 months, which is of sufficient length to enable the observation of clinically significant trends, and appropriate covariates were considered, including careful assessment of the $\mathrm{Z}$ line. Several shortcomings are also evident: Loss to follow-up was substantial, especially in the group with no IM, where almost half of patients did not have a repeat examination; also, given that an irregular $\mathrm{Z}$ line is not an indication for a repeat EGD, it is unclear whether 
these follow-up examinations were being done as part of the study protocol, or for clinical care. If the latter is true, the results must be viewed in a somewhat different light, due to the bias inherent in the motivations influencing patients to have repeat procedures: Patients who return for ongoing GERD symptoms might be expected to have a different prevalence of $\mathrm{BE}$ than those who are asymptomatic.

As with most studies of this type, the numbers with complete data are somewhat low to confidently draw the conclusions we would like to make. While the authors note that major complications did not develop in the 50 patients with IM with an irregular $Z$ line who were followed until the end of the study, would they still feel comfortable drawing this conclusion if a 51st patient developed EAC? Finally, it is unclear exactly which endoscopist performed the examinations, and whether any effort was made to ensure that the same endoscopist performed both examinations. Given the substantial inter-observer variability in the diagnosis of an irregular $\mathrm{Z}$ line [7], it is likely that different endoscopists might reach different conclusions regarding progression or regression of this lesion. Such variability almost certainly accounts for at least some proportion of the nine patients with incident BE (all, tellingly, short-segment BE), as well as the remarkably high $32 \%$ of initially abnormal patients who were noted to have a "normal" $\mathrm{Z}$ line on follow-up endoscopy. It would be instructive for a separate observer to review the before-and-after endoscopic photographs of these "normalized" cases, to see how much of the change was truly in the $Z$ line and how much of the change was due to the perception of the endoscopist.

The results of the present study add to a sparse, confusing, and inconclusive literature on the natural history of the irregular $\mathrm{Z}$ line (Table 1). Thota et al. performed a recent prospective multicenter study of 167 patients with an irregular $\mathrm{Z}$ line and IM. In a median follow-up of 71 months, none of the patients progressed to BE, dysplasia, or EAC [1]. In another population-based study by Jung et al. of 86 patients with an irregular Z line and IM, 2\% progressed to LGD and none progressed to develop BE, high-grade dysplasia (HGD), or EAC during the 8-year follow-up period [8]. Similarly, progression to neoplasia was also estimated to be low at $0.01 \%$ in another study of 204 patients with BE segment length $<1 \mathrm{~cm}$, which used population-based estimates to model annual cancer transition rates in patients with differing BE segment lengths [9].

The presence of a hiatal hernia can not only make it more difficult to accurately differentiate between an irregular $\mathrm{Z}$ line and short-segment BE on index endoscopic exam, but can also falsely increase the reported prevalence of IM if biopsies were obtained from the cardia due to sampling error. The authors attempted to address the issue of sampling error by only including biopsies obtained from the squamocolumnar junction that had evidence of both squamous tissue and columnar tissue. Nonetheless, one may take biopsies from a perfectly regular $\mathrm{Z}$ line including both squamous tissue and columnar tissue, and as a result not avert sampling of gastric cardia. Furthermore, a second pathologist reviewed all histologic samples with dysplasia. Despite this, in the initially IM-positive group, $63 \%$ were negative for IM on follow-up examination with biopsy. These results are similar to a previous study that reported that more than half of those with an irregular $\mathrm{Z}$ line with IM on index biopsy did not have IM on follow-up examination [1], underscoring the problem of sampling error that is magnified in the presence of very short segments of columnar mucosa. The presence of a hiatal hernia may also complicate the interpretation of the presence of $\mathrm{BE}$, in that over-insufflation of the esophagus
Table 1 Summary of studies showing progression to Barrett's esophagus (BE) and dysplasia with an irregular $\mathrm{Z}$ line or IM of the gastroesophageal junction

\begin{tabular}{|c|c|c|c|}
\hline Author & Itskoviz et al. & Thota et al. & Jung et al. \\
\hline Journal & DDS & Gastroenterology & AJG \\
\hline Year & 2018 & 2017 & 2011 \\
\hline Study type & Prospective & Prospective & Retrospective \\
\hline $\mathrm{Z}$ line definition & $\begin{array}{l}\text { Columnar } \\
\text { mucosa }<0.5 \mathrm{~cm}\end{array}$ & Columnar mucosa $<1 \mathrm{~cm}$ & $\begin{array}{l}\text { IM on biopsy } \\
\text { from GE } \\
\text { junction }\end{array}$ \\
\hline Patients $(n)$ & 166 & 167 & 86 \\
\hline Follow-up time, mos & 70 & 71 & 96 \\
\hline BE, $n$ & 9 & 0 & 0 \\
\hline $\mathrm{LGD}^{\mathrm{a}}, n$ & 2 & 0 & 2 \\
\hline $\mathrm{HGD}^{\mathrm{b}} / \mathrm{EAC}^{\mathrm{c}}, n$ & 0 & 0 & 0 \\
\hline Reversion to regular $\mathrm{Z}$ line & $32 \%$ & $53 \%$ & - \\
\hline
\end{tabular}

${ }^{a}$ Low-grade dysplasia

${ }^{\mathrm{b}}$ High-grade dysplasia

${ }^{\mathrm{c}}$ Esophageal adenocarcinoma 


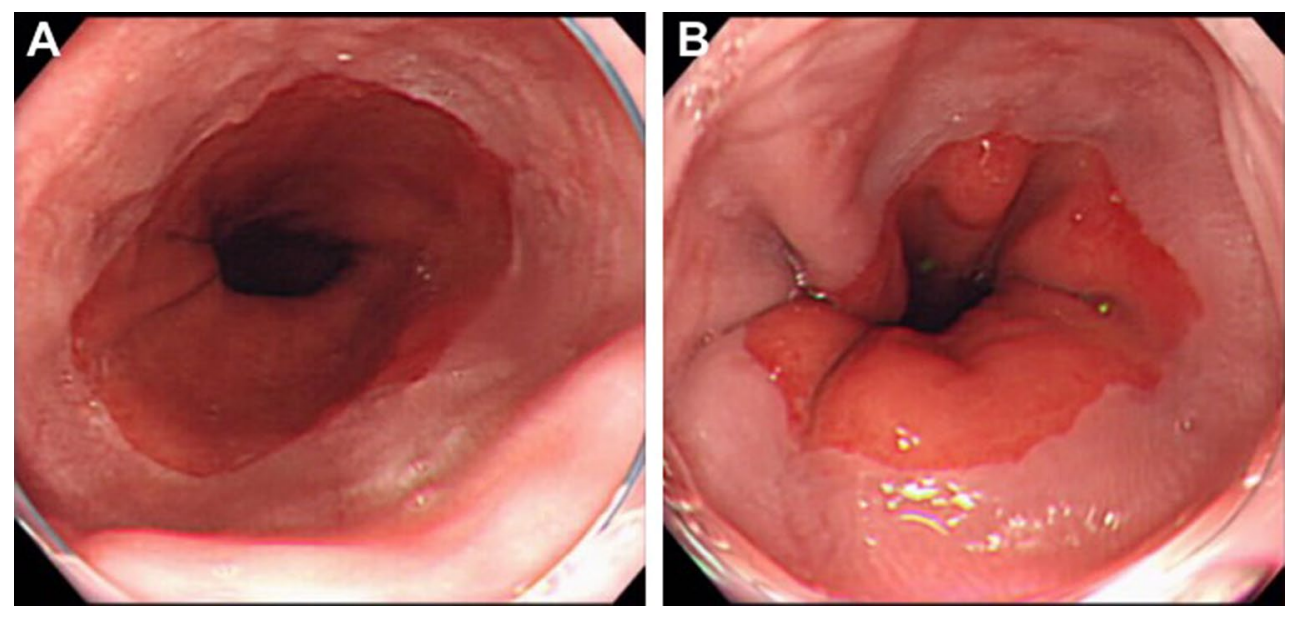

Fig. 1 Over-insufflation of the esophagus (a) can cause the hernia sac to have the appearance of Barrett's esophagus compared to the same esophagus without over-insufflation (b), in which the $\mathrm{Z}$ line appears to be regular (Image courtesy of Dr. Prateek Sharma)

may confound endoscopists by giving the hernia sac the appearance of a segment of BE (Fig. 1).

Even with all this confusion and potential for bias, the results from this study and the existing literature in balance show that the irregular $\mathrm{Z}$ line is a low-risk lesion. Current evidence does not support routine biopsies from an irregular $\mathrm{Z}$ line with an excursion $<1 \mathrm{~cm}$, since, as stated above, such biopsies may commit patients to lifelong surveillance examinations if IM is detected. We as gastroenterologists will likely continue to struggle to understand this problem, because we do not have accurate or reliable guidelines for the assessment, measurement, and reporting of columnar mucosa measuring $<1 \mathrm{~cm}$. While a four-grade classification system for assessing the $\mathrm{Z}$ line has been previously proposed that correlated with prevalence of IM [2], it has not been adopted and applied in the clinical setting. Until such time when superior measurements of minute changes around the $\mathrm{Z}$ line are possible or novel biomarkers are validated, endoscopists should recognize that small amounts of Barrett's, like beauty, are in the eye of the beholder. Currently, the limited data regarding the risk of neoplastic progression in those with an irregular $\mathrm{Z}$ line are such that an irregular $\mathrm{Z}$ line should not be routinely biopsied.

Funding Funding for this analysis was supported in part by NIH Award T32 DK07634 (SE), K24DK100548 (NJS).

\section{References}

1. Thota PN, Vennalaganti P, Vennelaganti S, et al. Low risk of highgrade dysplasia or esophageal adenocarcinoma among patients with barrett's esophagus less than $1 \mathrm{~cm}$ (irregular Z line) within 5 years of index endoscopy. Gastroenterology. 2017;152:987-992.

2. Wallner B, Sylvan A, Stenling R, Janunger KG. The esophageal Z-line appearance correlates to the prevalence of intestinal metaplasia. Scand J Gastroenterol. 2000;35:17-22.

3. Dickman R, Levi Z, Vilkin A, Zvidi I, Niv Y. Predictors of specialized intestinal metaplasia in patients with an incidental irregular Z line. Eur J Gastroenterol Hepatol. 2010;22:135-138.

4. Chalasani N, Wo JM, Hunter JG, Waring JP. Significance of intestinal metaplasia in different areas of esophagus including esophagogastric junction. Dig Dis Sci. 1997;42:603-607.

5. Shaheen NJ, Falk GW, Iyer PG, Gerson LB. ACG clinical guideline: diagnosis and management of Barrett's esophagus. Am J Gastroenterol. 2016;111:30-50.

6. Itskoviz D, Levi Z, Boltin D, et al. Risk of neoplastic progression among patients with an irregular $\mathrm{Z}$ line on long-term follow-up. Dig Dis Sci. (Epub ahead of print). https://doi.org/10.1007/s1062 0-018-4910-1.

7. Guda NM, Partington S, Vakil N. Inter- and intra-observer variability in the measurement of length at endoscopy: implications for the measurement of Barrett's esophagus. Gastrointest Endosc. 2004;59:655-658.

8. Jung KW, Talley NJ, Romero Y, et al. Epidemiology and natural history of intestinal metaplasia of the gastroesophageal junction and Barrett's esophagus: a population-based study. Am J Gastroenterol. 2011;106:1447-1455. (quiz 1456).

9. Pohl H, Pech O, Arash H, et al. Length of Barrett's oesophagus and cancer risk: implications from a large sample of patients with early oesophageal adenocarcinoma. Gut. 2016;65:196-201. 